\title{
Deep learning and manual assessment show that the absolute mitotic count does not contain prognostic information in triple negative breast cancer
}

\author{
Maschenka C. A. Balkenhol ${ }^{1}$ (D) $\cdot$ Peter Bult $^{1} \cdot$ David Tellez $^{1} \cdot$ Willem Vreuls $^{2} \cdot$ Pieter C. Clahsen $^{3} \cdot$ Francesco Ciompi $^{1}$ • \\ Jeroen A. W. M. van der Laak ${ }^{1}$
}

Accepted: 25 March 2019 / Published online: 15 April 2019

(C) The Author(s) 2019

\begin{abstract}
Purpose The prognostic value of mitotic count for invasive breast cancer is firmly established. As yet, however, limited studies have been aimed at assessing mitotic counts as a prognostic factor for triple negative breast cancers (TNBC). Here, we assessed the prognostic value of absolute mitotic counts for TNBC, using both deep learning and manual procedures.

Methods A retrospective TNBC cohort $(n=298)$ was used. The absolute manual mitotic count was assessed by averaging counts from three independent observers. Deep learning was performed using a convolutional neural network on digitized H\&E slides. Multivariable Cox regression models for relapse-free survival and overall survival served as baseline models. These were expanded with dichotomized mitotic counts, attempting every possible cut-off value, and evaluated by means of the c-statistic. Results We found that per $2 \mathrm{~mm}^{2}$ averaged manual mitotic counts ranged from 1 to 187 (mean 37.6, SD 23.4), whereas automatic counts ranged from 1 to 269 (mean 57.6; SD 42.2). None of the cut-off values improved the models' baseline c-statistic, for both manual and automatic assessments.

Conclusions Based on our results we conclude that the level of proliferation, as reflected by mitotic count, does not serve as a prognostic factor for TNBC. Therefore, TNBC patient management based on mitotic count should be discouraged.
\end{abstract}

Keywords Triple negative breast cancer $\cdot$ Mitotic count $\cdot$ Artificial intelligence $\cdot$ Prognosis

\section{Introduction}

Recent advances in machine learning have resulted in computer algorithms that are capable of analysing entirely digitized microscopic tissue sections (whole slide images; WSI). It has been shown that such algorithms can, for instance, accurately detect and delineate tumour areas in

Maschenka C. A. Balkenhol

maschenka.balkenhol@radboudumc.nl

1 Department of Pathology, Radboud University Medical Center, PO Box 9100, 6500, HB Nijmegen, the Netherlands

2 Department of Pathology, Canisius Wilhelmina Hospital, Nijmegen, the Netherlands

3 Department of Pathology, Haaglanden Medical Center, 's-Gravenhage, the Netherlands breast and colon tissue sections and detect mitotic figures in breast cancer [1-3]. Next to direct use in research and clinical practice, such algorithms are also of interest to reassess the diagnostic/prognostic value of widely used morphological criteria. As these algorithms allow a fully automatic analysis of large numbers of tissue sections with high reproducibility, this opens up new ways to establish 'evidence-based' pathology. In the present study we used deep learning to evaluate the prognostic value of mitosis counting for triple negative breast cancer (TNBC).

TNBCs comprise $\sim 15 \%$ of all breast tumours, and are characterized by absence of expression of the oestrogen receptor (ER) and the progesterone receptor (PR) and absence of overexpression of the human epidermal growth factor receptor 2 (HER2) [4]. TNBCs are known to occur at a relatively young age and to have a worse prognosis 
than their hormone receptor positive counterparts [5]. In routine pathology practice, every newly diagnosed invasive breast tumour will undergo histological grading [6-8]. Histological grading of breast cancer is routinely performed by means of the modified Bloom and Richardson grading system $[9,10]$, which comprises a three-tiered classification system. Microscopically assessed scores, expressing the severity of nuclear pleomorphism, the relative amount of tubule formation and the mitotic count in a $2 \mathrm{~mm}^{2}$ area are summed up and translated into an overall histological grade. The histological grade ranges from grade 1 , with features similar to normal breast epithelium, to grade 3 , being most deviant from normal breast epithelium.

The vast majority of TNBCs is of histological grade 3, while grade 1 TNBCs are rare [11-14]. It has been suggested, however, that histological grade does not provide prognostic information for TNBC [11, 15]. TNBC tumours display wide ranges of mitotic counts [16, 17], with most tumours showing counts that largely exceed the minimum number required for grade 3 of the modified Bloom and Richardson grading system. These tumours, therefore, often fall in the highest class for mitotic counting, not reflecting the wide variation in absolute mitotic counts in TNBC. To date, no studies have been reported assessing the prognostic value of mitotic counts in TNBC. In addition, considering the wide range of mitotic figures present in TNBC, it may be questioned whether the cut-off values of the modified Bloom and Richardson grading system are applicable to TNBC or whether better suited TNBC-specific cut-off values are available. The application of deep learning in the present study allows for a comprehensive analysis of absolute mitotic counts, even in the presence of very high densities of such cells.

Previously, we [15] developed a multivariable prognostic model for TNBC in which histological subtype was found to serve as an independent prognostic factor. In the present study, this existing multivariable model was used as a baseline to study the added prognostic value of mitotic count for TNBC. In addition to the 'grade 3' cut-off value of the modified Bloom and Richardson grading system, we investigated the prognostic value of a range of alternative mitotic count cut-off values. To be able to identify alternative cut-off values in the most objective manner, we performed currently used manual assessment and average counts made independently by three pathologists, as well as a counting procedure based on state-of-the-art deep learning strategies. Combined with whole slide scanning of microscopic tissue sections, deep convolutional neural networks (CNN) [18] have been shown to be highly suited for routinely performed pathological assessments, such as metastasis detection in breast cancer sentinel lymph nodes [19] and for mitosis detection [1].

\section{Materials and methods}

\subsection{Patients and tissue selections}

In a previous study, a multicentre retrospective cohort of TNBC was established using the Netherlands Comprehensive Cancer Registry (IKNL; a nationwide registry in which all malignancies in the Netherlands are registered) [15]. The cohort comprises 597 patients who were diagnosed with TNBC between the years 2006 and 2014 in the Eastern Netherlands in an academic hospital (Radboudumc, Nijmegen) or a general hospital (Canisius Wilhelmina Hospital, Nijmegen; Jeroen Bosch Hospital, 'sHertogenbosch; Bernhoven Hospital, Uden; Hospital Pantein, Boxmeer). Patients with stage IV disease on initial presentation and patients who were treated with neoadjuvant therapy were excluded. For each tumour, one representative tissue block was selected based on inspection of archival tissue sections for the presence of the tumour burden and the presence of a transition from tumour to normal breast tissue (the border of the tumour, often referred to as "invasive margin") [20]. From every selected tissue block, one new slide was cut and stained with H\&E in the Radboudumc pathology department according to routine practice. All tumours underwent central histopathological revision for histological subtype and grade (MCAB, $\mathrm{PB}$ ) using currently applicable guidelines [10,21].

For all patients, clinical and follow up data were retrieved from the Netherlands Comprehensive Cancer Registry (overall survival; OS) and from local patient files (relapse-free survival; RFS). The interval between the date of diagnosis of TNBC via core needle biopsy or fine needle aspiration and the date of clinically and/ or pathologically detected recurrence of TNBC was defined as RFS. The occurrence of hormone receptor and/ or HER2 positive breast cancer was regarded as a new primary tumour and not as a recurrence. If no recurrence occurred, patients were censored at the date of last follow up. OS was defined as the interval between the date of diagnosis of TNBC and the date of death or the moment of last follow up. The REMARK guidelines for reporting tumour marker prognostic studies were followed [22] and the study was conducted according 
to the Standards for Reporting of Diagnostic Accuracy (STARD) guideline [23].

\subsection{Ethical approval}

The requirement for ethical approval was waived by the institutional review board (case number 2015-1711) of the Radboudumc. All patient material and data were treated according to the Code of Conduct for the Use of Data in Health Research [24] and the Code of Conduct for responsible use of human tissue in the context of health research [25].

\subsection{Manual mitosis counting}

Three observers (MCAB: pathology resident; WV and PCC: pathologists with special interest in breast cancer) independently assessed the absolute mitotic counts for all tumours. All observers performed exhaustive visual mitosis counting in a $2 \mathrm{~mm}^{2}$ area, which was selected according to the modified Bloom and Richardson grading system $[9,10]$. The absolute number of mitoses was recorded, without translating into predefined classes. All observers were blinded for any clinical or pathological information, as well as for the scores of the other observers.

\subsection{Automatic mitosis counting}

For automatic mitosis counting we used a previously described deep learning algorithm [1]. In brief, algorithm training was performed using 18 tumours of the TNBC cohort, for which an additional H\&E slide was made and scanned on a Pannoramic 250 Flash II slide scanner (3DHistech, Hungary) at a spatial resolution of $0.25 \mu \mathrm{m} /$ pixel. Next, slides were de-stained and immunohistochemically restained using an anti-phosphorylated histone $\mathrm{H} 3$ (PHH3) antibody, which stains mitotic figures. [26]. PHH3-stained slides were scanned using the same scanner and resolution as for the H\&E slides. Resulting pairs of H\&E and PHH3 whole slide images (WSI) were subsequently co-registered (i.e., images were aligned such that there was a pixel level correspondence between two images). This procedure allowed exact localization of the $\mathrm{PHH} 3$ positive cells in the H\&E section (Fig. 1). Deep learning algorithms are typically trained using large sets of labelled examples. The set of images of mitotic figures resulting from the de-staining and restaining procedure described above provided a very extensive training set, producing a state-of-the-art algorithm for the detection of mitoses in $\mathrm{H} \& \mathrm{E}$ stained slides.

The H\&E slides of the TNBC cohort were scanned using the same Pannoramic 250 Flash II slide scanner and settings as the algorithm was trained on. The deep learning algorithm was applied to resulting H\&E WSI to automatically detect all mitotic figures (example in Fig. 2a, b). To establish an automated procedure for mitosis counting, comparable to manual counting, we calculated the number of mitoses detected by the deep learning algorithm in circles with a $2 \mathrm{~mm}^{2}$ area in every possible location in the WSI. Of all potential counting locations, the one with the highest mitotic density was automatically selected (Fig. 2b, c). The number of mitotic figures in this automatically identified hotspot was reported (automatic count; AMC).

For visual inspection, all automatically detected mitotic figures and the $2 \mathrm{~mm}^{2}$ circle were projected on the H\&E WSI (Fig. 2c). Because the algorithm does not discriminate between mitotic figures in benign and malignant epithelium, a number of cases with a low mitotic density in the invasive tumour area showed a $2 \mathrm{~mm}^{2}$ hotspot outside the tumour ( 52 of the 597 tumours). In these cases, the tumours were delineated by a pathology resident (MCAB) and the algorithm was applied again, now forced to designate the hotspot in the delineated area.

\subsection{Statistical analysis}

Because visual counting of mitoses is a very labourintensive procedure, we performed a power analysis to calculate the number of tumours needed to be included for manual mitosis counting. As no literature is available on the prognostic value of the mitotic count in TNBC, we based our power calculations on studies that have reported hazard ratio's for mitotic counts in the general breast cancer population [27-30]. The reported hazard ratio's in these studies varied between 1.5 and $>8$. For TNBC, we assume that the mitotic count yields a HR in the lower range of this spectrum. For the power analyses we, therefore, assumed a hazard ratio of 2.5. For an alpha significance level of 0.05 , a power of $80 \%$ and a risk of developing a recurrence within 5 years of $20 \%$ ( $19.6 \%$ in the present cohort), the required sample size for a HR of 2.5 was 292 . Using an overall risk of dying within 5 years of $25 \%$ ( $25.1 \%$ in the present cohort), the required sample size was 200 . Based on these power calculations, in the present study we selected $50 \%$ of the cases $(n=298)$ of the previously described cohort [15] as follows: after ranking all patients by incidence date (date of diagnosis with TNBC by either histology or cytology) every second patient was included. The distribution of variables of interest and of number of events between the selected and un-selected cases was compared using cross tabulation. No significant differences were observed ( $p>0.05$; data not 


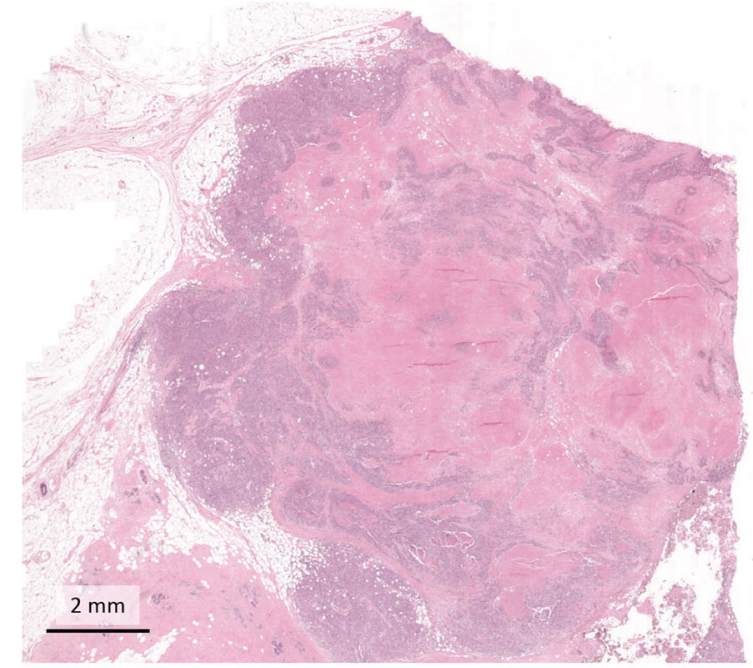

a

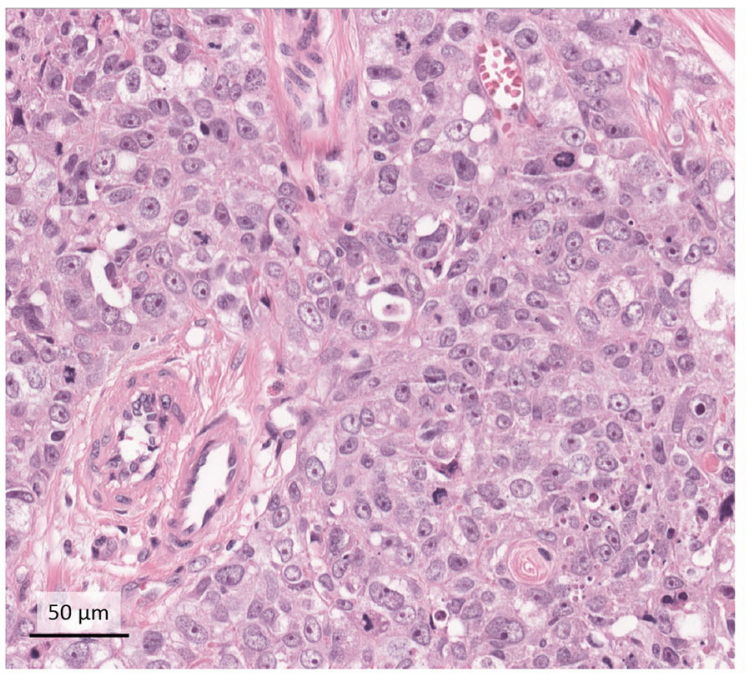

C

Fig. 1 One example of the 18 TNBC cases that was used to train the deep learning algorithm. (a) Overview at low magnification of the additional H\&E slide. After de-staining and re-staining using PHH3 (b), the

shown) using Pearson Chi-Square test. Also, independent sample $\mathrm{T}$ tests showed no significant differences $(p>0.05$; data not shown) in mean time to events between selected and un-selected cases. Interobserver variability between observers and between the $\mathrm{CNN}$ and observers was expressed as intraclass correlation coefficients (ICC). We used a 2-way random-effects model and tested for absolute agreement with a reliability calculated from a single measure (corresponding to $\operatorname{ICC}(2,1)$ according to the Shrout and Fleiss convention [31]). For every tumour, the mean manual mitotic count (MMC) was calculated as the

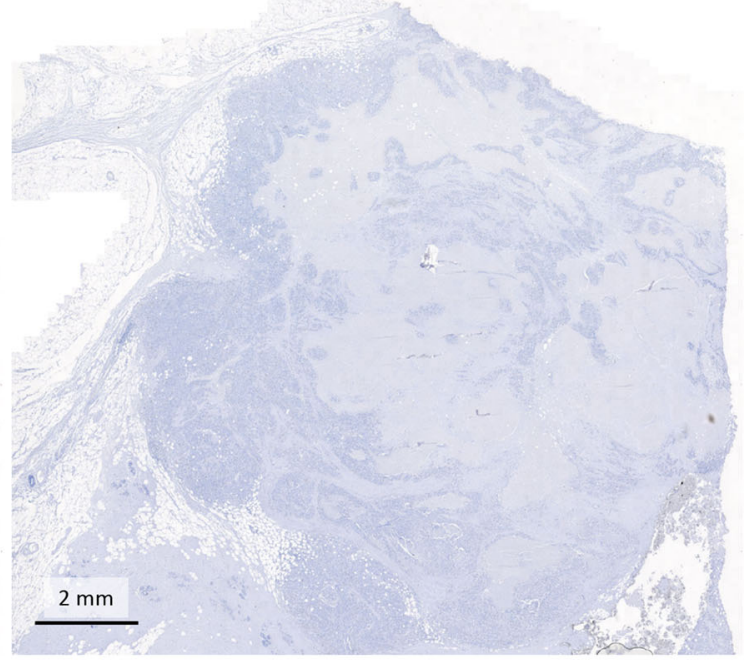

b

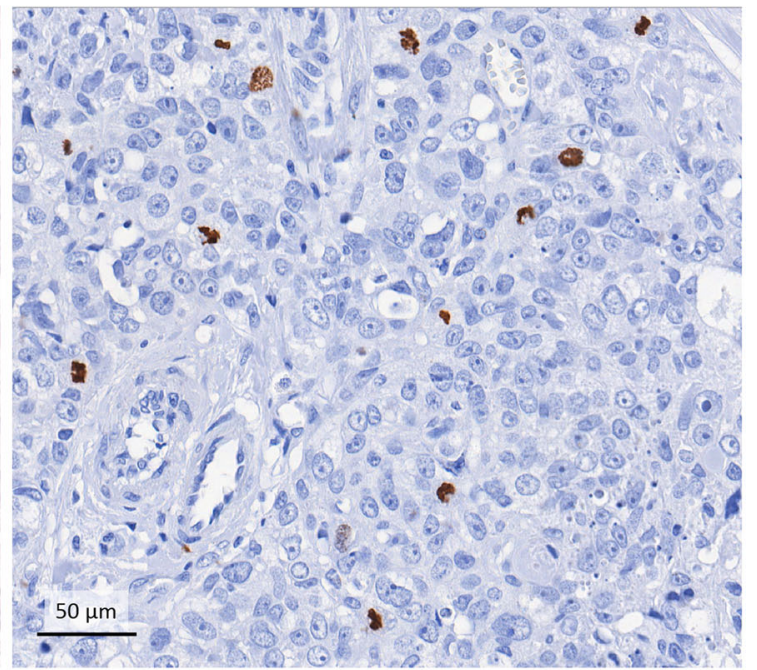

d

images could be co-registered to allow precise localization of the $\mathrm{PHH} 3$ positive cells in the H\&E section. (c) and (d) show exact correspondence between the $\mathrm{H} \& \mathrm{E}$ and $\mathrm{PHH} 3$ sections at high magnification

average over the three observers. Multivariable Cox regression analysis was performed to assess the prognostic value for a range of cut-off values for the MMC and the AMC, with RFS and OS as the primary outcome measures. Our previously described models [15] for RFS and OS of TNBC were used as baseline prognostic models. The baseline model consists of the variables age, primary tumour stage, regional lymph node stage, histological subtype, primary surgical treatment, adjuvant systemic therapy and adjuvant radiotherapy. Interaction terms between mitotic counts and available clinicopathological 


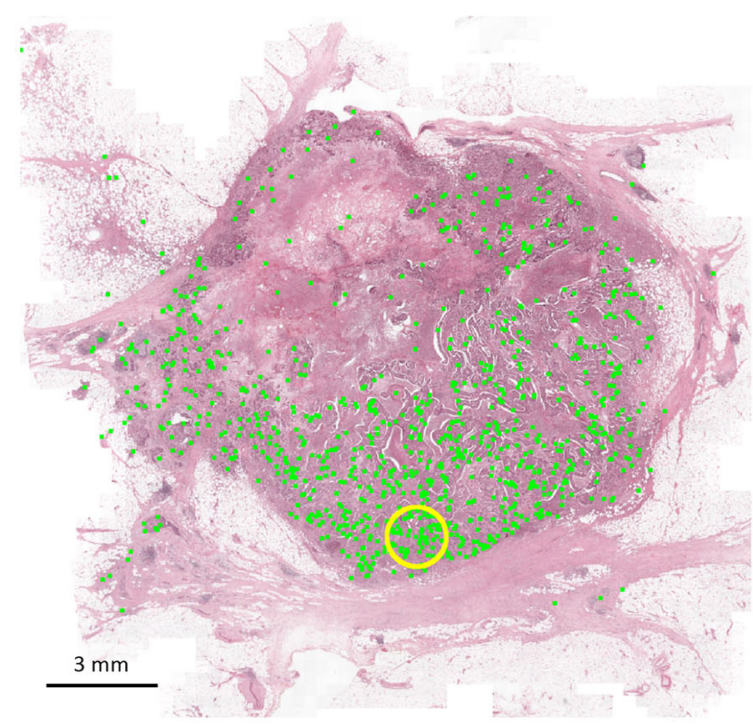

$\mathbf{a}$

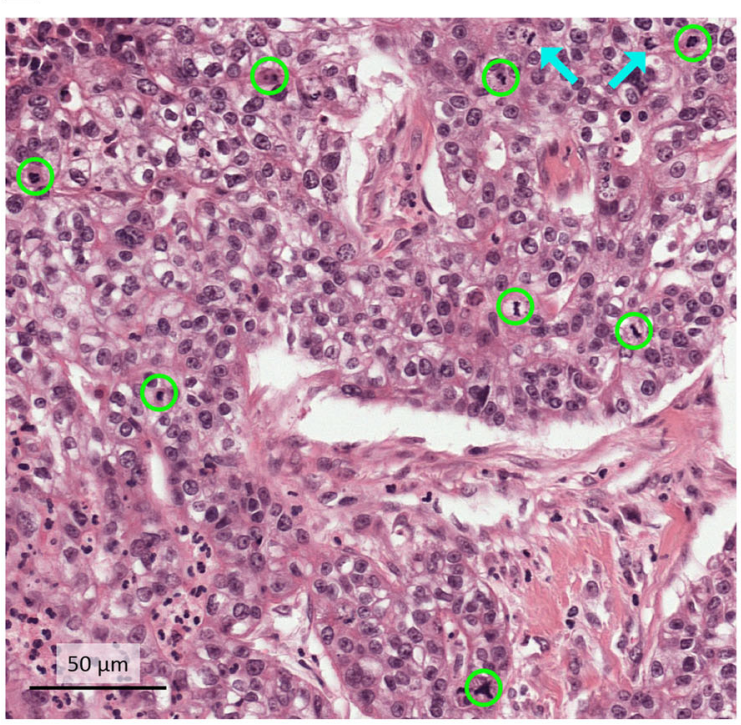

C

Fig. 2 Example of the deep learning algorithm applied to one of the TNBC tumours. (a) Overview at low magnification of the deep learning result. Every detected mitotic figure is marked with a green dot. The yellow circle indicates the $2 \mathrm{~mm}^{2}$ area with the highest density of mitotic figures. (b) Hotspot area as found by the deep learning

variables for RFS and OS were calculated to investigate whether the prognostic value of the mitotic count was different at different values of the other causal variable. We performed Cox analysis using the baseline model and separately adding the dichotomized MMC and AMC as a variable, for a range of different cut-off values. The lowest $10 \%$ and highest $10 \%$ of values of the MMC and the AMC

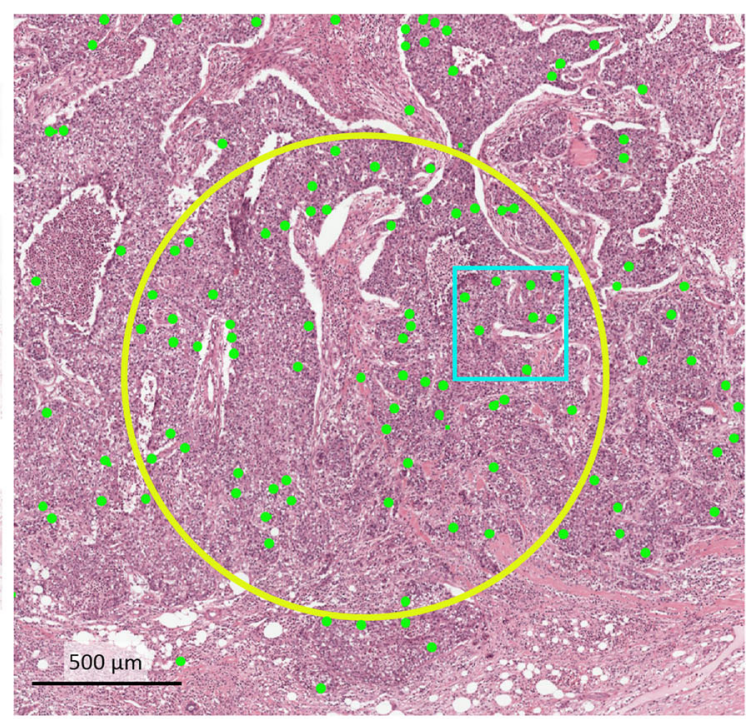

b

algorithm at higher magnification. The blue rectangle in the yellow hotspot circle relegates to the area that is shown in c. (c) Selected area (blue rectangle in $\mathrm{b}$ ) at high magnification in which the mitotic figures found by the deep learning algorithm are circled in green. Two mitotic figures are missed by the algorithm (blue arrows, top right)

were not considered as cut-off values. The range of values tested also included the cut-off value of 15 , which discriminates between the classes 2 and 3 of the modified Bloom and Richardson mitotic score [10].

As a performance measure for assessment of the mod$\mathrm{el}$, the c-statistic was used. The c-statistic indicates the discriminative power of a regression model. The approach 
Table 1 Overview of patient and tumour characteristics of the triple negative breast cancer cohort $(n=298)$

\begin{tabular}{|c|c|c|}
\hline & $n$ & $\%$ \\
\hline \multicolumn{3}{|l|}{ Gender } \\
\hline Female & 298 & 100.0 \\
\hline \multicolumn{3}{|l|}{ Age, years } \\
\hline$\geq 50$ & 193 & 64.8 \\
\hline$<50$ & 105 & 35.2 \\
\hline \multicolumn{3}{|l|}{ Primary tumour stage* } \\
\hline $\mathrm{T} 1$ & 161 & 54.0 \\
\hline $\mathrm{T} 2$ & 124 & 41.6 \\
\hline T3 & 10 & 3.4 \\
\hline $\mathrm{T} 4$ & 3 & 1.0 \\
\hline \multicolumn{3}{|l|}{ Regional lymph node stage* } \\
\hline N0 (including isolated tumour cells) & 206 & 69.1 \\
\hline N1 & 64 & 21.5 \\
\hline $\mathrm{N} 2$ & 14 & 4.7 \\
\hline N3 & 6 & 2.0 \\
\hline Nx (regional lymph nodes could not be assessed) & 8 & 2.7 \\
\hline \multicolumn{3}{|l|}{ Histological grade $[9,10]$} \\
\hline 1 & 1 & 0.3 \\
\hline 2 & 29 & 9.7 \\
\hline 3 & 268 & 89.9 \\
\hline \multicolumn{3}{|l|}{ Histological subtype [21] } \\
\hline Invasive carcinoma of no special type & 264 & 88.6 \\
\hline Special histological subtypes** & 34 & 11.4 \\
\hline \multicolumn{3}{|l|}{ Primary surgical treatment } \\
\hline Mastectomy & 106 & 35.6 \\
\hline Breast conserving surgery & 186 & 64.4 \\
\hline \multicolumn{3}{|l|}{ Adjuvant systemic therapy } \\
\hline None & 130 & 43.6 \\
\hline Anthracyclines & 69 & 23.2 \\
\hline Anthracyclines with taxanes & 97 & 32.6 \\
\hline Other regimes & 2 & 0.7 \\
\hline \multicolumn{3}{|l|}{ Adjuvant radiotherapy } \\
\hline No & 191 & 64.1 \\
\hline Yes & 107 & 35.9 \\
\hline \multicolumn{3}{|l|}{ Development of recurrence*** } \\
\hline No & 238 & 79.9 \\
\hline Yes & 60 & 20.1 \\
\hline \multicolumn{3}{|l|}{ Deceased (overall) } \\
\hline No & 222 & 74.5 \\
\hline Yes & 76 & 25.5 \\
\hline
\end{tabular}

*Primary tumour stage, regional lymph node stage and TNM stage are classified according to TNM 6th edition [34] for the years 2006 until 2009 and TNM 7th edition [35] that was in use from 2010. However, no changes considering the classification of the pathological T-stage and $\mathrm{N}$-stage were made in the TNM 7th edition, resulting in comparable stages between the 6 th and 7 th edition

**Metaplastic carcinoma (14 patients), invasive lobular carcinoma (6 patients), adenoid cystic carcinoma (4 patients), glycogen rich clear cell carcinoma ( 2 patients), malignant adenomyoepithelioma ( 2 patients), medullary carcinoma (1 patient), invasive carcinoma with osteoclast like giant cells ( 1 patient)

***The presence of a recurrence was confirmed either clinically (imaging studies) or with additional pathological examination

of Harrell et al. was used to calculate the c-statistic [32], which is the preferred approach for studies focusing on long term risk prediction and in which not all individuals experience the event of interest [33]. As an additional experiment, we analysed the results of AMC on the total cohort of 597 TNBC tumours, applying the procedure described above.

For all analyses, confidence intervals were set at the 95\% level and a minimal $p$ value of $<0.05$ was considered statistically significant. All analyses were performed using statistical software SPSS (version 24.0; IBM, Chicago, USA) and R (version 3.5.1).

\section{Results}

\subsection{Patient demographics and tumour characteristics of the triple negative breast cancer cohort}

Table 1 shows the patient and tumour characteristics of the selected cases. The majority of patients were 50 years or older at the time of diagnosis $(64.8 \%)$. About half of the tumours were smaller than $2 \mathrm{~cm}(54.0 \%)$ in size. The prevailing histological subtype was invasive carcinoma NST (88.6\%). Patients who were treated with chemotherapy were given anthracyclines with or without the addition of taxanes. For the group of patients treated with taxanes, no survival benefit was observed when compared to the anthracycline only patient group (data not shown). None of the patients were treated with the first generation chemotherapy regime CMF (cyclophosphamide, methotrexate, and 5-fluorouracil). In addition, no patients were treated with platinum salts. About one in five patients were confronted with a recurrence of TNBC (20.1\%) and one in four patients died during the follow up period $(25.5 \%)$. For the patients that developed a recurrence, the median time for developing a clinically detected recurrence was 28.8 months after primary TNBC diagnosis. The median time to TNBC-specific death was 3.7 months (mean 8.0 months) after being diagnosed with a recurrence of TNBC.

\subsection{Baseline prognostic model}

Baseline multivariable Cox regression analysis showed that a high primary tumour stage, a high regional lymph node stage and no administration of adjuvant therapy were correlated with a worse survival (Table 2). The values of the cstatistic for the baseline RFS model and the baseline OS model were 0.745 and 0.761 , respectively. 
Table 2 Baseline multivariable model for relapse-free survival and overall survival for the selected cases of the triple negative breast cancer cohort $(\mathrm{n}=$ 298)

$\begin{array}{lll}\text { Relapse free survival } & p \text { value } & \text { Overall survival } \\ \text { HR }(95 \% \mathrm{CI}) & & \text { HR }(95 \% \mathrm{CI})\end{array}$

Age, years

$$
<50
$$$$
\geq 50
$$

Primary tumour stage*

T1
T2
T3
T4
Regional lymph node stage*
N0 (including isolated tumour cells)
N1
N2
N3

Nx (regional lymph nodes could not be assessed)

Histological type [21]

Invasive carcinoma NST

Special histological subtypes

Primary surgical treatment

Mastectomy

Breast conserving surgery

Adjuvant systemic therapy

None

Anthracyclines

Anthracyclines with taxanes

Other regimes

Adjuvant radiotherapy

No

Yes

$$
\begin{aligned}
& 1 \text { (ref) } \\
& 0.63(0.32-1.24) \\
& 1 \text { (ref) } \\
& 1.58(0.89-2.82) \\
& 3.31(1.23-8.94) \\
& \text { NA }
\end{aligned}
$$

1 (ref)

$1.45(0.72-2.91)$

$9.16(3.62-23.17)$

7.53 (1.99-28.41)

NA

1 (ref)

$2.21(1.08-4.53)$

1 (ref)

$1.04(0.54-2.00)$

1 (ref)

$0.88(0.42-1.85)$

$0.52(0.23-1.19)$

$\mathrm{NA}$

1 (ref)

$0.56(0.27-1.17)$
0.179

1 (ref)

$0.72(0.35-1.46)$

0.359

1 (ref)

$2.85(1.66-4.89)$

$5.20(1.73-15.65)$

$<0.001$

0.018

NA

NA

0.003

NA

(ref)

0.296

$<0.001$

$1.46(0.79-2.70)$

0.225

$4.72(1.90-11.72)$

0.001

0.003

4.13 (0.93-18.30)

0.062

NA

NA

NA

1 (ref)

$1.37(0.69-2.70)$

0.369

1 (ref)

$0.93(0.52-1.67)$

0.819

0.916

1 (ref)

$0.32(0.15-0.67)$

0.002

$0.24(0.10-0.56)$

0.001

0.122

NA

NA

1 (ref)

$0.45(0.24-0.84)$

0.012

Abbreviations: $C I$ confidence interval, $H R$ hazard ratio, NA not applicable, NST no special type, ref.: reference category

* Primary tumour stage, regional lymph node stage and TNM stage are classified according to TNM 6th edition [34] for the years 2006 until 2009 and TNM 7th edition [35] that was in use from 2010. However, no changes considering the classification of the pathological T-stage and N-stage were made in the TNM 7th edition, resulting in comparable stages between the 6th and 7th TNM edition

\subsection{Prognostic value of the mitotic count and the value of alternative cut-off values}

The MMC ranged from 1 to 187 (median 34.7; mean 37.6; SD 23.4). The level of agreement between the three observers was good (intraclass correlation coefficient: 0.60 , range $0.585-0.616$ ). The upper and lower boundaries used for the range of cut-off values for the manual count were set at 66 and 12, respectively. The AMC ranged from 1 to 269 (median 50.5; mean 57.6; SD
42.2). The level of agreement (ICC) between AMC and observers ranged from 0.497 to 0.626 . For the automatic count, the upper and lower boundaries of the cut-off values were set to 110 and 12 . Figure 3 shows the discriminative capacity (expressed in the c-statistic) of the baseline prognostic model (red line) and the baseline prognostic model with the mitotic count as additional variable (green line) for both MMC (left) and AMC (right). For RFS (Fig. 3a-b), the c-statistic value of the baseline prognostic model was found to be nearly equal 


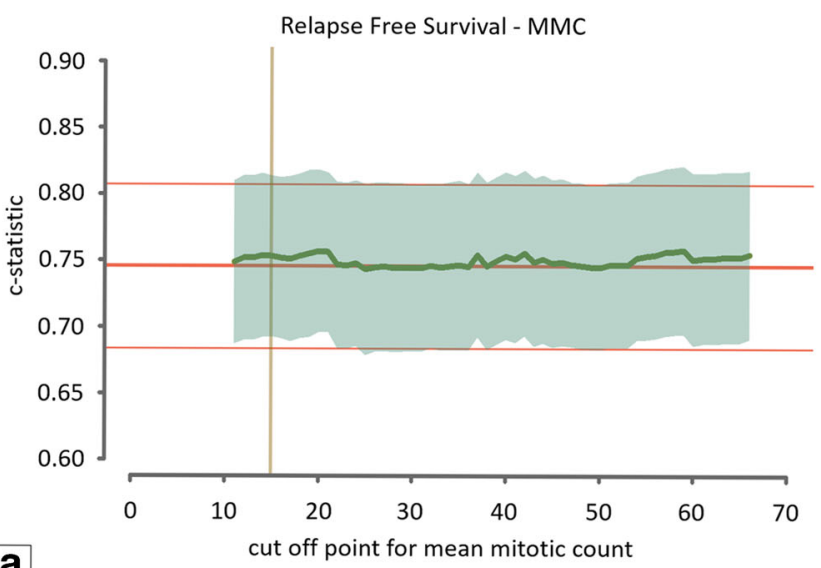

a

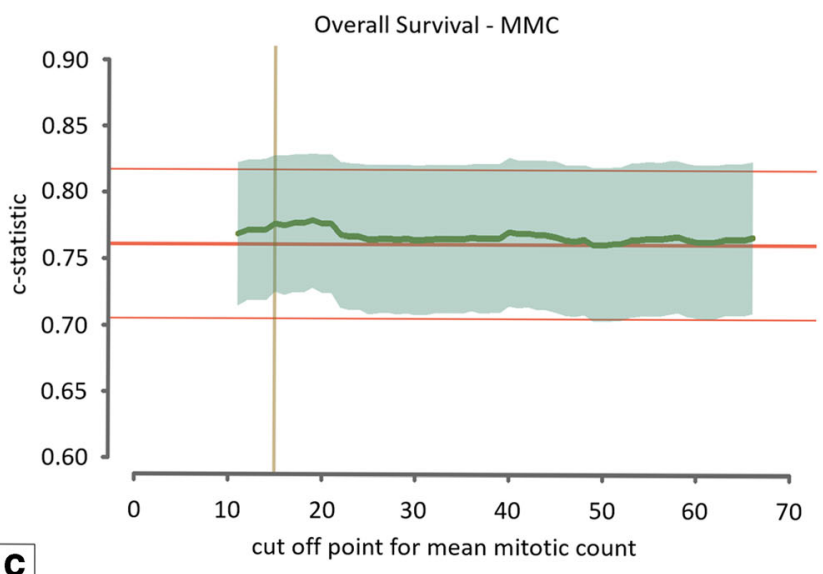

C

Fig. 3 Graphic representation of the c-statistic value of the Cox regression model. The middle red line represents the value of the $\mathrm{c}-$ statistic of the model without the mitotic count (baseline model) with corresponding upper and lower 95\% confidence interval indicated by the fine red lines. The green line indicates the value of the c-statistic for every cut-off value of the mean mitotic score for the range between 12 and 66 mitoses per $2 \mathrm{~mm}^{2}$ for the averaged manual assessments and between 12 and 110 mitoses per $2 \mathrm{~mm}^{2}$ for the automatic assessment.

to the model with the addition of the mitotic count for all possible cut-off values, indicating that the mitotic count does not improve the baseline model. In addition, for OS (Fig. 3c-d) the curve that corresponds with the value of the c-statistic of the model with the addition of the mitotic count was found to fluctuate with very small margins around the curve of the c-statistic value of the baseline model. The algorithm was additionally applied to the unselected cases of the TNBC cohort using the automatic counts of the total cohort $(n=597)$ as input for the multivariable Cox regression model. Application of AMC on
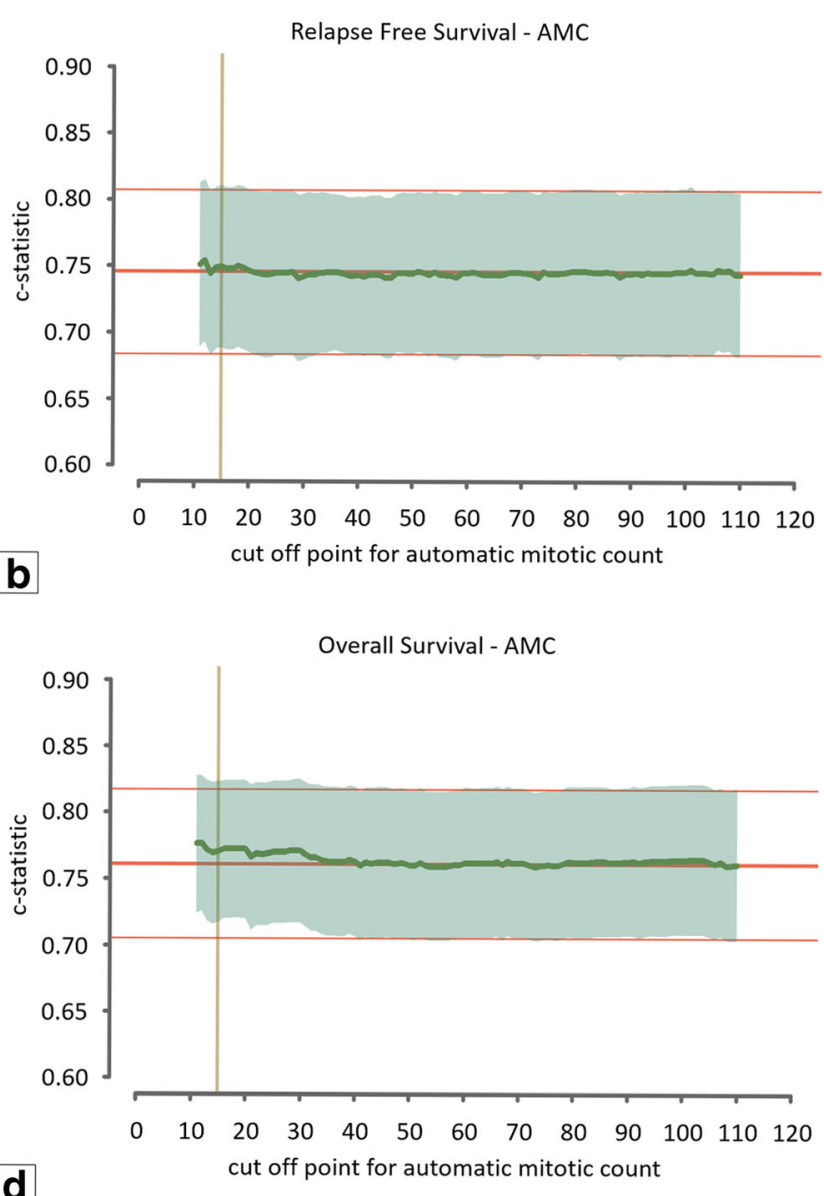

The translucent green area indicates the $95 \%$ upper and lower confidence interval for the value of the c-statistic. The vertical yellow line indicates the cut-off value of the modified Bloom and Richardson mitotic score (15 mitoses per $2 \mathrm{~mm}^{2}$ ). (a) Relapse-free survival for the mean mitotic count of the manual assessments. (b) Relapse-free survival for the mitotic count of the automatic assessment. (c) Overall survival for the mean mitotic count of the manual assessments. (d) Overall survival for the mitotic count of the automatic assessment

the extended cohort did not show any additional prognostic value of the mitotic count (Fig. 4).

\subsection{Subgroup analysis of the prognostic value of the mitotic count}

The relation between the mitotic counts and the available clinicopathological variables were calculated using interaction terms. The only variable for which interaction with the mitotic count was found was type of adjuvant systemic therapy. Figures 5 and 6 visualise the baseline 


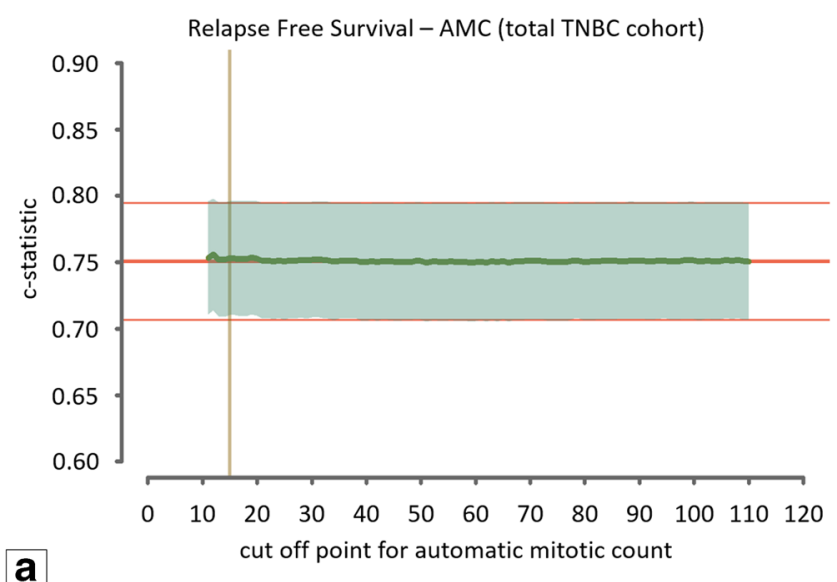

Fig. 4 Graphic representation of the c-statistic value of the Cox regression model for the total TNBC cohort $(n=597)$ in combination with the automatic assessment of the mitotic count. The middle red line represents the value of the c-statistic of the model without the mitotic count (baseline model) with corresponding upper and lower 95\% confidence interval indicated by the fine red lines. The green line

c-statistics and additional prognostic value of the mitotic counts for the different adjuvant systemic therapy regimes. Due to too small numbers, no calculation for the patients who received other systemic therapy regimens $(n=2)$ could be performed. Figures $5 \mathrm{a}$ and $6 \mathrm{a}$ show that for a selection of cut-off values, the c-statistic of the model that includes the mitotic count (green line) exhibits higher values than the baseline c-statistic. No cut-off value, however, exhibited values outside the confidence intervals of the baseline models (fine red lines).

\section{Discussion}

Previously, we showed that histological subtype is an independent prognostic feature for RFS in TNBC [15]. In the present study we explored the prognostic value of the mitotic count for TNBC. The multivariable Cox regression model developed in our prior study served as baseline model and was used to assess the putative added prognostic value of the mitotic count for TNBC. The Cox regression models for RFS and OS were expanded with the mitotic count, after which we analysed the discriminative value of the regression model for a wide range of cut-off values for the mitotic count. We found that the addition of the mitotic count did not

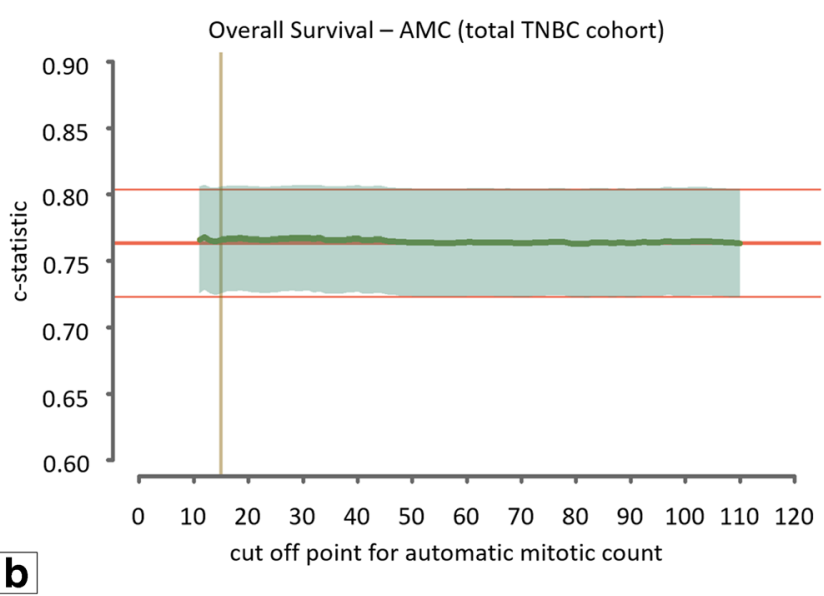

indicates the value of the c-statistic for every cut-off value of the mean mitotic score for the range between 12 and 110 mitoses per $2 \mathrm{~mm}^{2}$. The translucent green area indicates the $95 \%$ upper and lower confidence interval for the value of the c-statistic. The vertical yellow line indicates the cut-off value of the modified Bloom and Richardson mitotic score (15 mitoses per $2 \mathrm{~mm}^{2}$ ). (a) Relapse-free survival. (b) Overall survival

improve the prognostic model for RFS or OS for any of the tested cut-off values.

TNBC tumours are characterized by the absence of ER and PR expression and the absence of HER2 overexpression. As such, the TNBC category contains a mixture of tumours with widely varying histopathological and genomic characteristics and, consequently, variable clinical courses of the disease. This situation poses challenges for the treating clinician, as the basis for therapy choices is small. Well-known prognostic factors are not yet established for TNBC. Recent work has focused on identifying immune-based and molecular features of TNBC to obtain prognostic [36, 37] and predictive [38-40] biomarkers. For the TNBC subtype, only scarce literature exists focusing on the prognostic value of proliferation. It has been shown that TNBC tumours express high levels of proliferative activity on the mRNA [41] and protein levels [42]. However, so far no strong proliferation-based prognosticator specifically for this type of cancer has evolved. We previously found that histopathological subtype serves as an independent prognostic factor, potentially identifying a small subgroup (up to $30 \%$ [11-13]) of TNBC with a worse prognosis. The present study shows that mitotic counting does not yield any prognostic information for TNBC. To the best of our knowledge, this is the first study that focused on the prognostic value of absolute 
Relapse Free Survival - MMC

No adjuvant systemic therapy

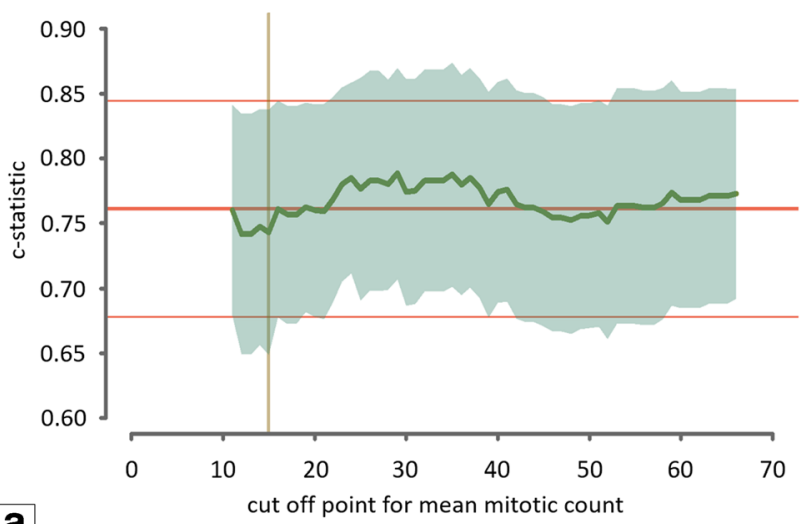

a
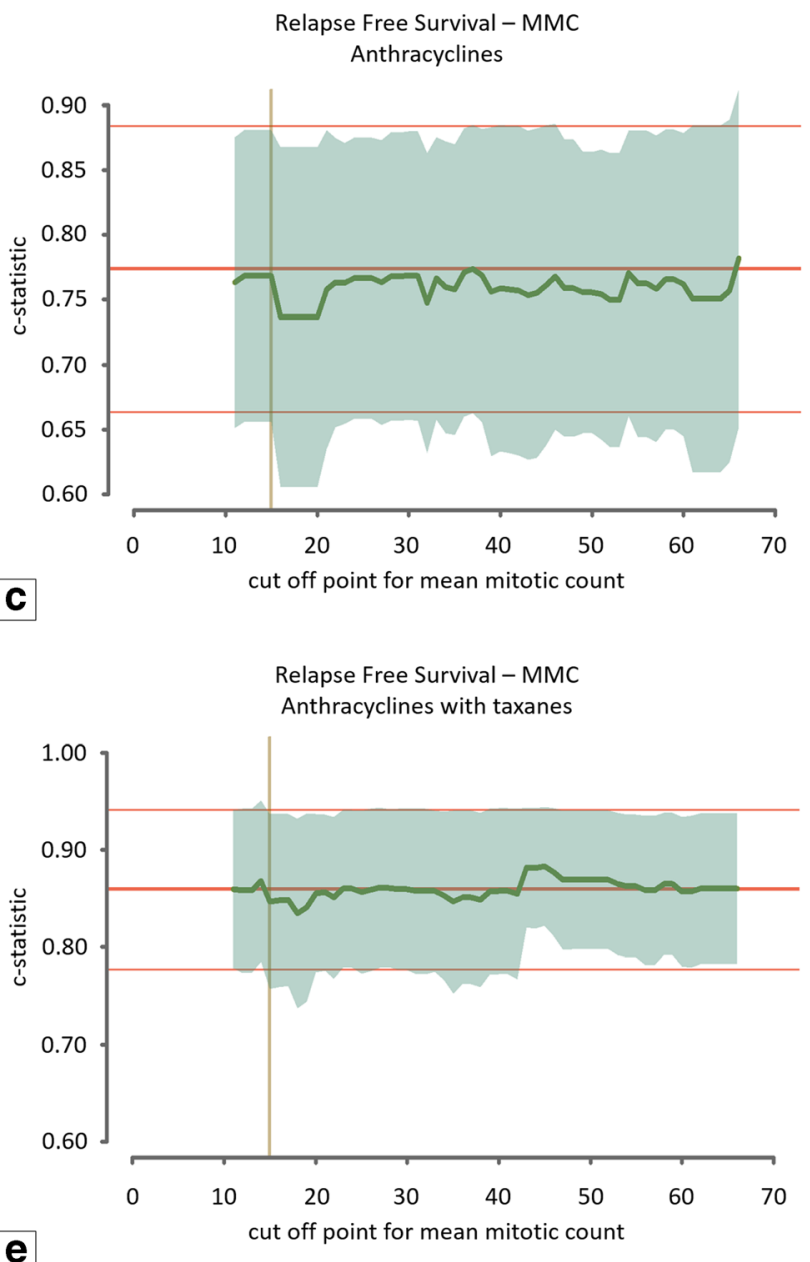

Relapse Free Survival - AMC

No adjuvant systemic therapy

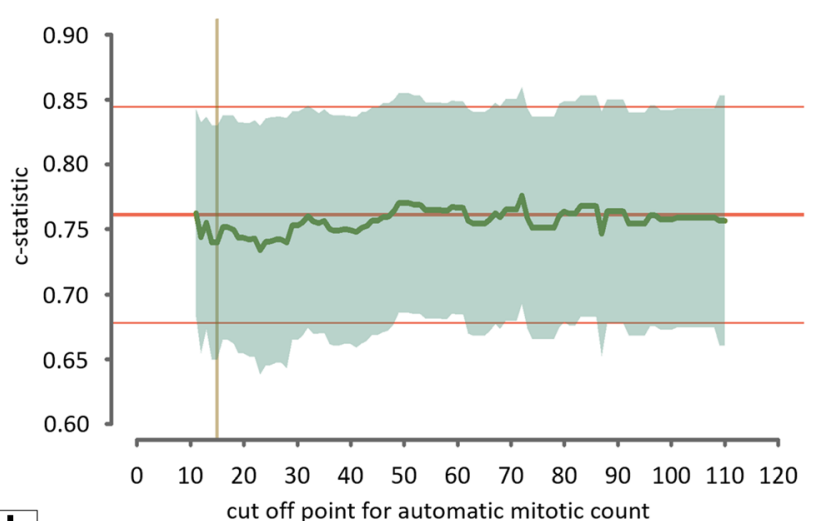

b
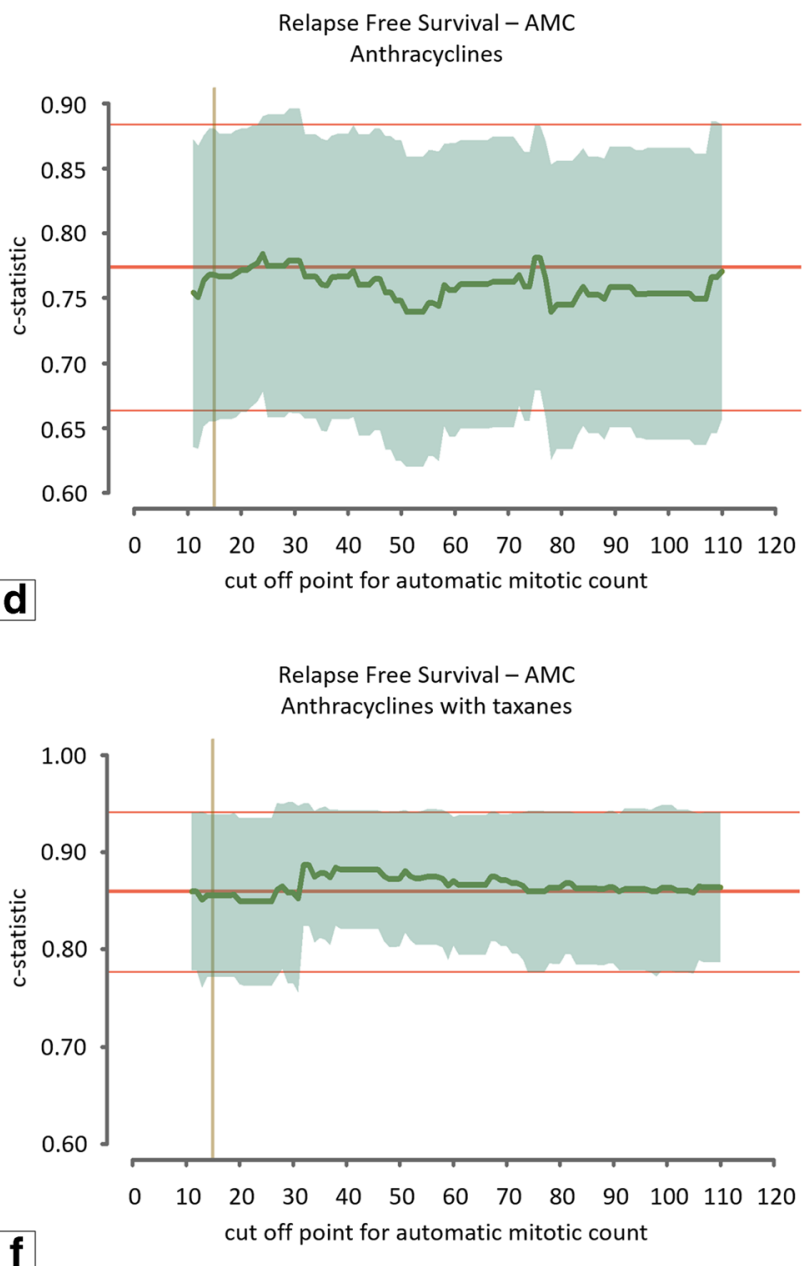
Fig. 5 Graphic representation of the c-statistic value of the Cox regression model for the different adjuvant systemic therapy regimens; relapse-free survival. The middle red line represents the value of the c-statistic of the model without the mitotic count (baseline model) with corresponding upper and lower $95 \%$ confidence interval indicated by the fine red lines. The green line indicates the value of the c-statistic for every cut-off value of the mean mitotic score for the range between 12 and 66 mitoses per $2 \mathrm{~mm}^{2}$ for the averaged manual assessments and between 12 and 110 mitoses per $2 \mathrm{~mm}^{2}$ for the automatic assessment. The translucent green area indicates the $95 \%$ upper and lower confidence interval for the value of the c-statistic. The vertical yellow line indicates the cut-off value of the modified Bloom and Richardson mitotic score (15 mitoses per $2 \mathrm{~mm}^{2}$ ). (a) Mean mitotic count of the manual assessments, patients who did not receive adjuvant systemic therapy ( $n=130)$. (b) Mitotic count of the automatic assessment, patients who did not receive adjuvant systemic therapy $(n=130)$. (c) Mean mitotic count of the manual assessments, patients who received anthracycline-based chemotherapy regimens $(n=69)$. (d) Mitotic count of the automatic assessment, patients who received anthracycline-based chemotherapy regimens $(n=69)$. (e) Mean mitotic count of the manual assessments, patients who received anthracycline with taxane-based chemotherapy regimens ( $n=97$ ). (f) Mitotic count of the automatic assessment, patients who received anthracycline with taxane-based chemotherapy regimens $(n=97)$

mitotic counts for TNBC. Mitotic counting as part of tumour grading is considered an established prognostic factor for breast cancer in general, and is routinely performed for every new case. Results from the present study indicate that we may have to re-consider the current practice of grading TNBC. Clearly, alternative prognostic factors for TNBC are urgently needed.

Although many studies have assessed the prognostic value of the mitotic count for invasive breast cancer, the prognostic value of the absolute mitotic count is not commonly studied. The mitotic count is typically analysed as a categorical variable that comprises three classes (low, middle and high) with cut-off values that are based on the modified Bloom and Richardson grading system [43, 44]. An exception is the study design of Kronqvist et al. [45, 46]. The prognostic value of a range of thresholds of the mitotic count was analysed by these authors in a cohort of 364 breast cancer patients. An optimal cut-off value for the mitotic count was determined by examining Chi-square values of the $\log$ rank test for every cut-off value for different subgroups of patients. Although this approach bears similarities with our study design, the most important difference is that our current study explores the prognostic value of cut-off values specifically for TNBC, which are known to exhibit much higher mitotic counts than general breast cancers. Also, we performed a multivariable approach to correct for other prognostic factors, in contrast to the univariable analyses applied by Kronqvist et al.

It has been shown that counting of mitotic figures is prone to subjectivity [42] due to a lack of standardization
[47, 48] and, additionally, is hampered by external factors such as fixation artefacts [49]. To become less dependent on individual assessments of the number of mitotic figures and of the selection of the area where to count, three observers independently assessed the mitotic count for every tumour in our study. We used the average of the three observers as the mean mitotic count for every tumour. By taking the average of the individual counts the interobserver variation was reduced. In addition, we applied a deep learning algorithm to assess the mitotic counts in an objective and reproducible manner. Automated counting based on deep learning potentially yields a more objective and reproducible measure [50]. Also, because of the extensive effort associated with manual counting of larger numbers of mitoses, only by using automated counting we could analyse the prognostic value in the entire cohort of almost 600 cases, adding considerable power to the study. The use of a deep learning-based algorithm in the present study showcases the potential of such techniques for re-evaluating existing histopathological features.

Next to research use, deep learning will most likely also find clinical application in histopathology. Algorithms such as the one used in the present study may be very useful in a clinical setting, pre-analysing scanned tissue sections before the pathologist starts the diagnostic process. With the introduction of whole slide imaging devices, the introduction of such algorithms will be strongly facilitated. An algorithm that has already processed WSI can subsequently be used to assist pathologists to reduce observer biases, and increase accuracy and efficiency. The first study on the potential of such a setup in a prospective setting showed that screening for metastases in sentinel lymph nodes of breast cancer patients was both faster and more accurate when a pathologist was assisted by a deep learningbased system [51].

This study has several strengths. Because our TNBC cohort was deduced from 5 different hospitals over several years, it can be regarded as a good reflection of the diverse TNBC population. Counting mitotic figures in $\mathrm{H} \& \mathrm{E}$ slides is a laborious and time-consuming task. The time and effort that the three observers have put into counting the absolute numbers of mitotic figures in nearly 300 tumours is, therefore, very valuable. The additional analyses of mitotic counts assessed by a computer-based algorithm, which showed comparable prognostic value as the counts of the human observers did, underscore the conclusion that mitotic count is of no prognostic value for TNBC. As stated, no previous reports have been published studying the prognostic value of mitotic counts in TNBC. Our analysis is limited by the constraints of a retrospective study, although we 


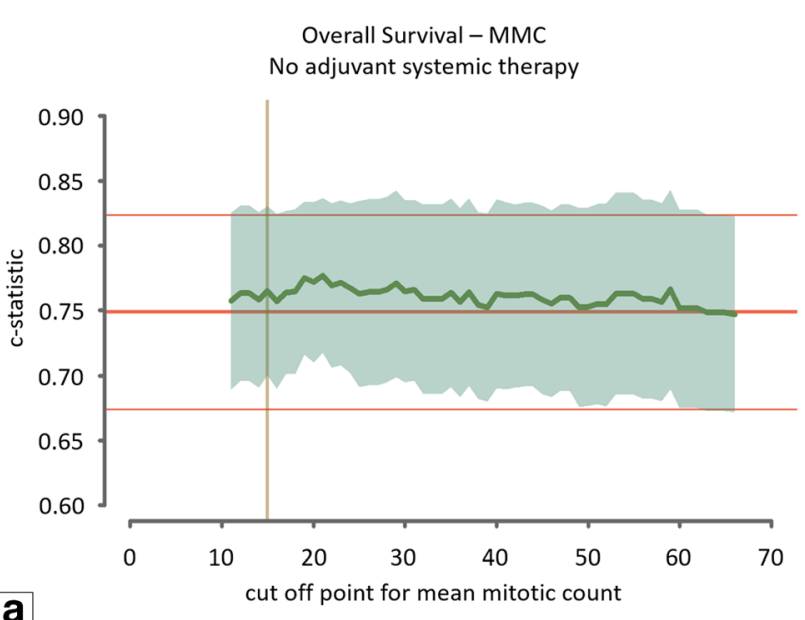

a
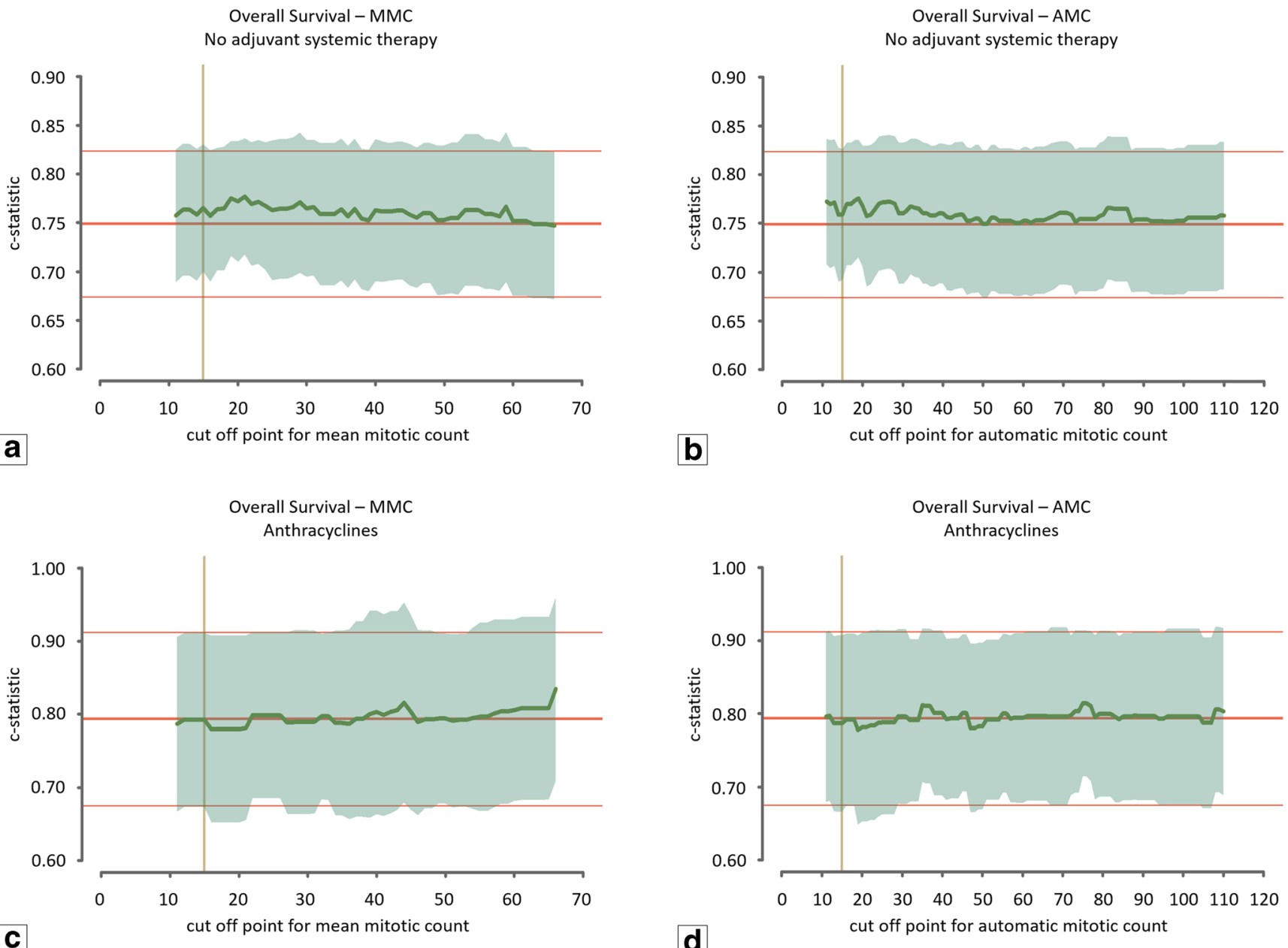

C

Overall Survival - MMC

Anthracyclines with taxanes

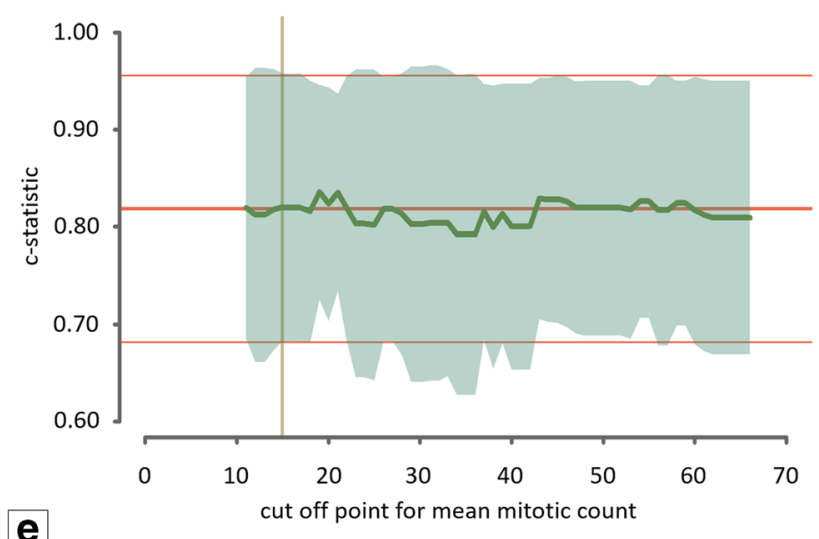

b

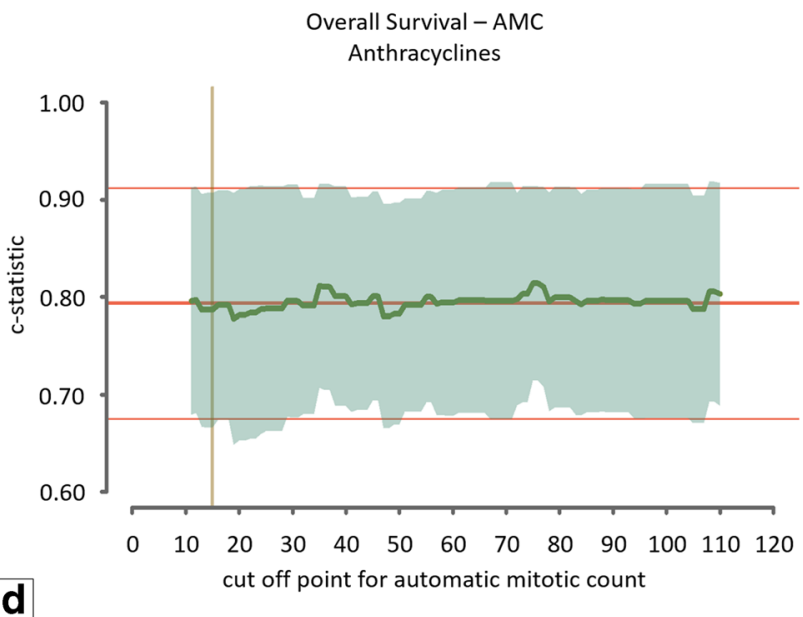

d

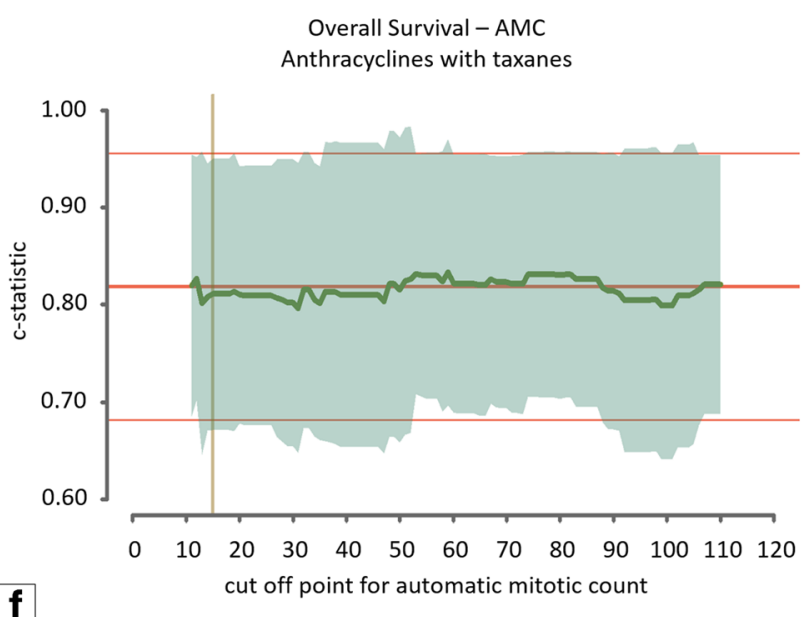


Fig. 6 Graphic representation of the c-statistic value of the Cox regression model for the different adjuvant systemic therapy regimens; overall survival. The middle red line represents the value of the c-statistic of the model without the mitotic count (baseline model) with corresponding upper and lower $95 \%$ confidence interval indicated by the fine red lines. The green line indicates the value of the c-statistic for every cut-off value of the mean mitotic score for the range between 12 and 66 mitoses per $2 \mathrm{~mm}^{2}$ for the averaged manual assessments and between 12 and 110 mitoses per $2 \mathrm{~mm}^{2}$ for the automatic assessment. The translucent green area indicates the $95 \%$ upper and lower confidence interval for the value of the c-statistic. The vertical yellow line indicates the cut-off value of the modified Bloom and Richardson mitotic score (15 mitoses per $2 \mathrm{~mm}^{2}$ ). (a) Mean mitotic count of the manual assessments, patients who did not receive adjuvant systemic therapy $(n=130)$. (b) Mitotic count of the automatic assessment, patients who did not receive adjuvant systemic therapy $(n=130)$. (c) Mean mitotic count of the manual assessments, patients who received anthracycline-based chemotherapy regimens $(n=69)$. (d) Mitotic count of the automatic assessment, patients who received anthracycline-based chemotherapy regimens $(n=69)$. (e) Mean mitotic count of the manual assessments, patients who received anthracycline with taxane-based chemotherapy regimens $(n=97)$. (f) Mitotic count of the automatic assessment, patients who received anthracycline with taxane-based chemotherapy regimens $(n=97)$

made a considerable effort to obtain high quality and complete follow up data by using a highly reliable nationwide cancer registry and an extensive review of the patient files. In conclusion, by analysing a wide range of cut-off values, we show that mitotic count does not improve the prognostic value of currently available multivariable prognostic models for RFS and OS for TNBC. Our results suggest that mitotic count is of no prognostic value for TNBC patients. As this is the first study that specifically explored the prognostic value of mitotic counts for TNBC, these results must be confirmed in independent TNBC cohorts. In addition, this study shows the potential of deep learning-based algorithms for evaluating histopathological features in large series in an objective manner.

Acknowledgements The authors would like to thank the registration team of the Netherlands Comprehensive Cancer Organisation (IKNL) for the collection of data from the Netherlands Cancer Registry.

Author's contribution MCAB, JAWMvdL and PB designed the study. MCAB (under supervision of $\mathrm{PB}$ ), WV and PCC performed the manual mitotic counting of the tumours. MCAB and JAWMvdL performed the statistical analyses. DT and FC developed the deep learning algorithm to detect mitotic figures in WSI of H\&E slides and analysed all tumours in the cohort with this algorithm. MCAB and JAWMvdL had full access to all study data and take responsibility for the integrity of the data, the accuracy of the data analysis, and interpretation of data. All authors were responsible for critical revisions, and all authors read and approved the final version of this manuscript.

\section{Compliance with ethical standards}

Conflict of interest Jeroen van der Laak is member of the scientific advisory boards of Philips, the Netherlands and ContextVision, Sweden and receives remuneration for product development for Philips, the Netherlands. The other authors have no conflicts of interest to disclose. This study was funded by a Junior Researcher grant from the Radboud University Medical Center Institute for Health Sciences (RIHS).

Open Access This article is distributed under the terms of the Creative Commons Attribution 4.0 International License (http:// creativecommons.org/licenses/by/4.0/), which permits unrestricted use, distribution, and reproduction in any medium, provided you give appropriate credit to the original author(s) and the source, provide a link to the Creative Commons license, and indicate if changes were made.

\section{References}

1. D. Tellez, M. Balkenhol, I. Otte-Holler, R. van de Loo, R. Vogels, P. Bult, C. Wauters, W. Vreuls, S. Mol, N. Karssemeijer, G. Litjens, J. van der Laak, F. Ciompi, Whole-slide mitosis detection in H\&E breast histology using $\mathrm{PHH} 3$ as a reference to train distilled staininvariant convolutional networks. Trans. Med. Imaging 28, 2126$2136(2018)$

2. O.G.F. Geessink, A. Baidoshvili, J.M. Klaase, B. Ehteshami Bejnordi, G.J.S. Litjens, G.W. van Pelt, W.E. Mesker, I.D. Nagtegaal, F. Ciompi, J.A.W.M. van der Laak, Computer aided quantification of intratumoral stroma yields an independent prognosticator in rectal cancer. Cell. Oncol. (2019). https://doi.org/10. 1007/s13402-019-00429-Z

3. Z. Guo, H. Liu, H. Ni, X. Wang, M. Su, W. Guo, K. Wang, T. Jiang, Y. Qian, A fast and refined cancer regions segmentation framework in whole-slide breast pathological images. Sci. Rep. 9, 882 (2019)

4. K.D. Voduc, M.C. Cheang, S. Tyldesley, K. Gelmon, T.O. Nielsen, H. Kennecke, Breast cancer subtypes and the risk of local and regional relapse. J. Clin. Oncol. 28, 1684-1691 (2010)

5. W.D. Foulkes, I.E. Smith, J.S. Reis-Filho, Triple-negative breast cancer. N. Engl. J. Med. 363, 1938-1948 (2010)

6. Netherlands Comprehensive Cancer Registry. Guid. Breast Cancer http://www.oncoline.nl. Accessed 25 May 2018

7. I.O. Ellis, Programmes NHSCS, Royal College of pathologists, the science C. Pathology reporting of breast disease: A joint document incorporating the third edition of the NHS breast screening programme's guidelines for pathology reporting in breast cancer screening and the second edition of the Royal College of pathologists' minimum dataset for breast cancer histopathology. London: NHS Cancer screening Programmes, Royal College of Pathologists, 2016

8. P.L. Fitzgibbons, College of American Pathologists, Protocol for the Examination of Specimens from Patients with Invasive Carcinoma of the Breast. Version InvasiveBreast 4.0.0.0. Protocol Posting Date: June 2017

9. H.J.G. Bloom, W.W. Richardson, Histological grading and prognosis in breast cancer: A study of 1409 cases of which 359 have been followed for 15 years. Br. J. Cancer 11, 359-377 (1957)

10. C.W. Elston, I.O. Ellis, Pathological prognostic factors in breast cancer. I. The value of histological grade in breast cancer: 
Experience from a large study with long-term follow-up. Histopathology. 19, 403-410 (1991)

11. R.A. Leon-Ferre, M.Y. Polley, H. Liu, J.A. Gilbert, V. Cafourek, D.W. Hillman, A. Elkhanany, M. Akinhanmi, J. Lilyquist, A. Thomas, V. Negron, J.C. Boughey, M.C. Liu, J.N. Ingle, K.R. Kalari, F.J. Couch, D.W. Visscher, M.P. Goetz, Impact of histopathology, tumor-infiltrating lymphocytes, and adjuvant chemotherapy on prognosis of triple-negative breast cancer. Breast Cancer Res. Treat. 167, 89-99 (2018)

12. S.A.M. Urru, S. Gallus, C. Bosetti, T. Moi, R. Medda, E. Sollai, A. Murgia, F. Sanges, G. Pira, A. Manca, D. Palmas, M. Floris, A.M. Asunis, F. Atzori, C. Carru, M. D'Incalci, M. Ghiani, V. Marras, D. Onnis, M.C. Santona, G. Sarobba, E. Valle, L. Canu, S. Cossu, A. Bulfone, P.C. Rocca, M.R. De Miglio, S. Orrù, Clinical and pathological factors influencing survival in a large cohort of triplenegative breast cancer patients. BMC Cancer 18, 56 (2018)

13. E. Montagna, P. Maisonneuve, N. Rotmensz, G. Cancello, M. Iorfida, A. Balduzzi, V. Galimberti, P. Veronesi, A. Luini, G. Pruneri, L. Bottiglieri, M.G. Mastropasqua, A. Goldhirsch, G. Viale, M. Colleoni, Heterogeneity of triple-negative breast cancer: Histologic subtyping to inform the outcome. Clin. Breast Cancer 13, 31-39 (2013)

14. F.C. Geyer, F. Pareja, B. Weigelt, E. Rakha, I.O. Ellis, S.J. Schnitt, J.S. Reis-Filho, The spectrum of triple-negative breast disease: High- and low-grade lesions. Am. J. Pathol. 187, 2139-2151 (2017)

15. M.C.A. Balkenhol, W. Vreuls, C.A.P. Wauters, S.J. Mol, J.A.W.M. van der Laak, P. Bult, Histological subtype is an independent prognostic feature of triple negative breast cancer (Submitted)

16. L.A. Carey, C.M. Perou, C.A. Livasy, L.G. Dressler, D. Cowan, K. Conway, G. Karaca, M.A. Troester, C.K. Tse, S. Edmiston, S.L. Deming, J. Geradts, M.C. Cheang, T.O. Nielsen, P.G. Moorman, H.S. Earp, R.C. Millikan, Race, breast cancer subtypes, and survival in the Carolina breast Cancer study. JAMA. 295, 2492-2502 (2006)

17. L. Zhang, X.I. Wang, S. Zhang, Tumor-infiltrating lymphocyte volume is a better predictor of neoadjuvant therapy response and overall survival in triple-negative invasive breast Cancer. Hum. Pathol. 80, 47-54 (2018)

18. Y. LeCun, Y. Bengio, G. Hinton, Deep learning. Nature. 521, 436444 (2015)

19. B.E. Bejnordi, M. Veta, P.J. van Diest, B. van Ginneken, N. Karssemeijer, G. Litjens, J.A.W.M. van der Laak, the CAMELYON16 Consortium, M. Hermsen, Q.F. Manson, M. Balkenhol, O. Geessink, N. Stathonikos, M.C. van Dijk, P. Bult, F. Beca, A.H. Beck, D. Wang, A. Khosla, R. Gargeya, H. Irshad, A. Zhong, Q. Dou, Q. Li, H. Chen, H.J. Lin, P.A. Heng, C. Haß, E. Bruni, Q. Wong, U. Halici, M.Ü. Öner, R. Cetin-Atalay, M. Berseth, V. Khvatkov, A. Vylegzhanin, O. Kraus, M. Shaban, N. Rajpoot, R. Awan, K. Sirinukunwattana, T. Qaiser, Y.W. Tsang, D. Tellez, J. Annuscheit, P. Hufnagl, M. Valkonen, K. Kartasalo, L. Latonen, P. Ruusuvuori, K. Liimatainen, S. Albarqouni, B. Mungal, A. George, S. Demirci, N. Navab, S. Watanabe, S. Seno, Y. Takenaka, H. Matsuda, H.A. Phoulady, V. Kovalev, A. Kalinovsky, V. Liauchuk, G. Bueno, M.M. Fernandez-Carrobles, I. Serrano, O. Deniz, D. Racoceanu, R. Venâncio, Diagnostic assessment of deep learning algorithms for detection of lymph node metastases in women with breast cancer. JAMA 318, 2199-2210 (2017)

20. S. Hendry, R. Salgado, T. Gevaert, P.A. Russell, T. John, B. Thapa, M. Christie, K. van de Vijver, M.V. Estrada, P.I. Gonzalez-Ericsson, M. Sanders, B. Solomon, C. Solinas, G.G.G.M. van den Eynden, Y. Allory, M. Preusser, J. Hainfellner, G. Pruneri, A. Vingiani, S. Demaria, F. Symmans, P. Nuciforo, L. Comerma, E.A. Thompson, S. Lakhani, S.R. Kim, S. Schnitt, C. Colpaert, C. Sotiriou, S.J. Scherer, M. Ignatiadis, S. Badve, R.H. Pierce, G.
Viale, N. Sirtaine, F. Penault-Llorca, T. Sugie, S. Fineberg, S. Paik, A. Srinivasan, A. Richardson, Y. Wang, E. Chmielik, J. Brock, D.B. Johnson, J. Balko, S. Wienert, V. Bossuyt, S. Michiels, N. Ternes, N. Burchardi, S.J. Luen, P. Savas, F. Klauschen, P.H. Watson, B.H. Nelson, C. Criscitiello, S. O'Toole, D. Larsimont, R. de Wind, G. Curigliano, F. André, M. LacroixTriki, M. van de Vijver, F. Rojo, G. Floris, S. Bedri, J. Sparano, D. Rimm, T. Nielsen, Z. Kos, S. Hewitt, B. Singh, G. Farshid, S. Loibl, K.H. Allison, N. Tung, S. Adams, K. Willard-Gallo, H.M. Horlings, L. Gandhi, A. Moreira, F. Hirsch, M.V. Dieci, M. Urbanowicz, I. Brcic, K. Korski, F. Gaire, H. Koeppen, A. Lo, J. Giltnane, M.C. Rebelatto, K.E. Steele, J. Zha, K. Emancipator, J.W. Juco, C. Denkert, J. Reis-Filho, S. Loi, S.B. Fox, Assessing tumorinfiltrating lymphocytes in solid tumors: A practical review for pathologists and proposal for a standardized method from the international immunooncology biomarkers working group: Part 1: Assessing the host immune response, TILs in invasive breast carcinoma and ductal carcinoma in situ, metastatic tumor deposits and areas for further research. Adv. Anat. Pathol. 24, 235-251 (2017)

21. S. R. Lakhani, I. O. Ellis, S. J. Schnitt, P. H. Tan, M. J. van de Vijver (eds.), World Health Organization Classification of Tumours. Classification of Tumours of the Breast, 4th edn. (IARC Press, Lyon, 2012)

22. L.M. McShane, D.G. Altman, W. Sauerbrei, S.E. Taube, M. Gion, G.M. Clark, Statistics subcommittee of the NCI-EORTC working group on Cancer diagnostics, reporting recommendations for tumor marker prognostic studies (REMARK). J. Natl. Cancer Inst. 97, 1180-1184 (2005)

23. P.M. Bossuyt, J.B. Reitsma, D.E. Bruns, C.A. Gatsonis, P.P. Glasziou, L. Irwig, J.G. Lijmer, D. Moher, D. Rennie, H.C. de Vet, H.Y. Kressel, N. Rifai, R.M. Golub, D.G. Altman, L. Hooft, D.A. Korevaar, J.F. Cohen, STARD group, STARD 2015: An updated list of essential items for reporting diagnostic accuracy studies. BMJ. 351, h5527 (2015)

24. Foundation Federation of Dutch Medical Scientific Societies (Federa). Code Of Conduct For Medical Research. 2004. https:// www.federa.org/codes-conduct. Accessed 2 May 2018

25. Foundation Federation of Dutch Medical Scientific Societies (Federa). Human tissue and Medical Research: Code of Conduct for Responsible Use. 2011. https://www.federa.org/codes-conduct. Accessed 2 May 2018

26. M.J. Hendzel, Y. Wei, M.A. Mancini, A. Van Hooser, T. Ranalli, B.R. Brinkley, D.P. Bazett-Jones, C.D. Allis, Mitosis-specific phosphorylation of histone $\mathrm{H} 3$ initiates primarily within pericentromeric heterochromatin during $\mathrm{G} 2$ and spreads in an ordered fashion coincident with mitotic chromosome condensation. Chromosoma. 106, 348-360 (1997)

27. P. Bult, P. Manders, H.M.P.M. Straatman, V.C.G. Tjan-Heijnen, L.V.A.M. Beex, J. Hendriks, J.W. Leer, A.L.M. Verbeek, R. Holland, In primary breast cancer the mitotic activity yields similar prognostic information as the histological grade: A study with longterm follow-up. Breast Cancer Res. Treat. 122, 77-86 (2010)

28. P. Manders, P. Bult, C.G.J. Sweep, V.C.G. Tjan-Heijnen, L.V.A.M. Beex, The prognostic value of the mitotic activity index in patients with primary breast cancer who were not treated with adjuvant systemic therapy. Breast Cancer Res. Treat. 77, 77-84 (2003)

29. J.P. Baak, P.J. van Diest, F.J. Voorhorst, E. van der Wall, L.V. Beex, J.B. Vermorken, E.A. Janssen, Prospective multicenter validation of the independent prognostic value of the mitotic activity index in lymph node-negative breast cancer patients younger than 55 years. J. Clin. Oncol. 23, 5993-6001 (2005)

30. E.A. Rakha, M. Aleskandarani, M.S. Toss, A.R. Green, G. Ball, I.O. Ellis, L.W. Dalton, Breast cancer histologic grading using digital microscopy: Concordance and outcome association. J. Clin. Pathol. 71, 680-686 (2018) 
31. T.K. Koo, M.Y. Li, A guideline of selecting and reporting intraclass correlation coefficients for reliability research. J Chiropr. Med. 15, $155-163(2016)$

32. F.E. Harrell, K.L. Lee, D.B. Mark, Tutorial in biostatistics: Multivariable prognostic models: Issues in developing models, evaluating assumptions and adequacy, and measuring and reducing errors. Stat. Med. 15, 361-387 (1996)

33. M.J. Pencina, R.B. D'Agostino, L. Song, Quantifying discrimination of Framingham risk functions with different survival C statistics. Stat. Med. 31, 1543-1553 (2012)

34. F. L. Greene, D. L. Page, I. D. Fleming, A. G. Fritz, C. M. Balch, D. G. Haller, M. Morrow (eds.), AJCC Cancer Staging Manual, 6th edn. (Springer, New York, 2002)

35. S. B. Edge, D. R. Byrd, C. C. Compton, A. G. Fritz, F. Greene, A. Trotti (eds.), AJCC Cancer Staging Manual, 7th edn. (Springer, New York, 2010)

36. H. Mori, M. Kubo, R. Nishimura, T. Osako, N. Arima, Y. Okumura, M. Okido, M. Yamada, M. Kai, J. Kishimoto, T. Miyazaki, Y. Oda, T. Otsuka, M. Nakamura, BRCAness as a biomarker for predicting prognosis and response to anthracycline-based adjuvant chemotherapy for patients with triple-negative breast cancer. PLoS One 11, e0167016 (2016)

37. N. Patel, D. Weekes, K. Drosopoulos, P. Gazinska, E. Noel, M. Rashid, H. Mirza, J. Quist, F. Brasó-Maristany, S. Mathew, R. Ferro, A.M. Pereira, C. Prince, F. Noor, E. Francesch-Domenech, R. Marlow, E. de Rinaldis, A. Grigoriadis, S. Linardopoulos, P. Marra, A.N.J. Tutt, Integrated genomics and functional validation identifies malignant cell specific dependencies in triple negative breast cancer. Nat. Commun. 9, 1044 (2018)

38. E.A. Mittendorf, A.V. Philips, F. Meric-Bernstam, N. Qiao, Y. Wu, S. Harrington, X. Su, Y. Wang, A.M. Gonzalez-Angulo, A. Akcakanat, A. Chawla, M. Curran, P. Hwu, P. Sharma, J.K. Litton, J.J. Molldrem, G. Alatrash, PD-L1 expression in triplenegative breast cancer. Cancer Immunol. Res. 2, 361-370 (2014)

39. N. Vidula, H.S. Rugo, Translating the molecular message of triplenegative breast cancer into targeted therapy. Clin. Cancer Res. 21, 1511-1513 (2015)

40. M. O'Loughlin, X. Andreu, S. Bianchi, E. Chemielik, A. Cordoba, G. Cserni, P. Figueiredo, G. Floris, M.P. Foschini, P. Heikkilä, J. Kulka, I. Liepniece-Karele, P. Regitnig, A. Reiner, A. Ryska, A. Sapino, A. Shalaby, E.S. Stovgaard, C. Quinn, E.M. Walsh, V. Zolota, S.A. Glynn, G. Callagy, Reproducibility and predictive value of scoring stromal tumour infiltrating lymphocytes in triplenegative breast cancer: A multi-institutional study. Breast Cancer Res. Treat. 171, 1-9 (2018)

41. B.P. Schneider, E.P. Winer, W.D. Foulkes, J. Garber, C.M. Perou, A. Richardson, G.W. Sledge, L.A. Carey, Triple-negative breast cancer: Risk factors to potential targets. Clin. Cancer Res. 14, 8010-8018 (2008)
42. Y. Pan, Y. Yuan, G. Liu, Y. Wei, P53 and Ki-67 as prognostic markers in triple-negative breast cancer patients. PLoS One 12, e0172324 (2017)

43. K. Beelen, M. Opdam, T. Severson, R. Koornstra, A. Vincent, J. Wesseling, J. Sanders, J. Vermorken, P. van Diest, S. Linn, Mitotic count can predict tamoxifen benefit in postmenopausal breast cancer patients while Ki67 score cannot. BMC Cancer 18, 761 (2018)

44. J.M. Chang, A.E. McCullough, A.C. Dueck, H.E. Kosiorek, I.T. Ocal, T.K. Lidner, R.J. Gray, N. Wasif, D.W. Northfelt, K.S. Anderson, B.A. Pockaj, Back to basics: Traditional Nottingham grade mitotic counts alone are significant in predicting survival in invasive breast carcinoma. Ann. Surg. Oncol. 22(Suppl 3), S509S515 (2015)

45. P. Kronqvist, T. Kuopio, Y. Collan, Morphometric grading in breast cancer: Thresholds for mitotic counts. Hum. Pathol. 29, 1462-1468 (1998)

46. P. Kronqvist, T. Kuopio, Y. Collan, Quantitative thresholds for mitotic counts in histologic grading: Confirmation in nonfrozen samples of invasive ductal breast cancer. Ann. Diagn. Pathol. 4, 65-70 (2000)

47. P. Boiesen, P.O. Bendahl, L. Anagnostaki, H. Domanski, E. Holm, I. Idvall, S. Johansson, O. Ljungberg, A. Ringberg, G. Ostberg, M. Fernö, Histologic grading in breast cancer: Reproducibility between seven pathologic departments. South Sweden breast Cancer group. Acta Oncol. 39, 41-45 (2000)

48. H.F. Frierson, R.A. Wolber, K.W. Berean, D.W. Franquemont, M.J. Gaffey, J.C. Boyd, D.C. Wilbur, Interobserver reproducibility of the Nottingham modification of the Bloom and Richardson histologic grading scheme for infiltrating ductal carcinoma. Am. J. Clin. Pathol. 103, 195-198 (1995)

49. E. Bergers, I. Jannink, P. van Diest, M.A. Cuesta, S. Meyer, J.C. van Mourik, J.P. Baak, The influence of fixation delay on mitotic activity and flow cytometric cell cycle variables. Hum. Pathol. 28, 95100 (1997)

50. M. Veta, P.J. van Diest, M. Jiwa, S. Al-Janabi, J.P. Pluim, Mitosis counting in breast cancer: Object-level interobserver agreement and comparison to an automatic method. PLoS One 11, e0161286 (2016)

51. D.F. Steiner, R. MacDonald, Y. Liu, P. Truszkowski, J.D. Hipp, C. Gammage, F. Thng, L. Peng, M.C. Stumpe, Impact of deep learning assistance on the histopathologic review of lymph nodes for metastatic breast cancer. Am. J. Surg. Pathol. 42, 1636-1646 (2018)

Publisher's note Springer Nature remains neutral with regard to jurisdictional claims in published maps and institutional affiliations. 